\title{
Utilidad de la laparoscopía en el tratamiento de abscesos post apendicectomía. Casos clínicos (video)
}

Usefulness of laparoscopy in the treatment of post-appendectomy abscesses. Clinical cases (video)

Javier Chinelli ${ }^{1}$ Rodrigo Hernández ${ }^{2}$ Gustavo Rodríguez Temesio ${ }^{3}$.

DOI: $10.31837 /$ cir.urug/3.2.7

Recibido: 4 de diciembre de 2018

Aceptado: 20 de diciembre de 2018

\section{Introducción}

Los abscesos residuales post-apendicectomía aparecen en un $4 \%$ en las formas simples y hasta en el $20 \%$ de las complicadas. El tratamiento puede ser conservador mediante antibioticoterapia, aunque los mayores de 4-5 cm requerirán drenaje, que puede ser percutáneo (de elección) o quirúrgico (laparotómico o laparoscópico). El abordaje laparoscópico permite una exploración completa de la cavidad abdomino-pélvica y el tratamiento de uno o varios abscesos, con menor dolor postoperatorio e infecciones parietales en comparación al abordaje laparotómico.

\section{Descripción de contenidos}

Caso 1: mujer, 19 años, apendicectomía laparoscópica (edematosa). Al $7^{0}$ día dolor abdominal, taquicardia y fiebre, leucocitosis elevada. TC: absceso retrocecal de $6 \mathrm{~cm}$. Se realiza drenaje laparoscópico completo y sin incidentes.

Caso 2: hombre, 32 años, apendicectomía laparoscópica (gangrenosa). Ileo postoperatorio, taquicardia y polipnea, TC al $4^{\circ}$ día: absceso del Douglas y leve cantidad de líquido libre intraabdominal. Se realiza drenaje laparoscópico de absceso del Douglas y toilette peritoneal. 
1,2,3 . Clínica Quirúrgica “2” Hospital Maciel, Facultad de Medicina, Universidad de la República, Montevideo, Uruguay jchinelli@hotmail.com 